\title{
Émergence des « learning analytics » en éducation : quelques défis et enjeux de l'analyse de l'apprentissage
}

\section{Emergence of Learning Analytics in Education: Challenges and Issues of Learning Analysis}

Séverine Parent, Université du Québec à Rimouski

Monique Baron, LIP6 - Sorbonne Université

\section{Résumé}

Lors d'EDUsummIT2019, un groupe de travail a mené une réflexion sur l'analyse de l'apprentissage (learning analytics). En tant que membres francophones de ce groupe, nous présentons et discutons dans cet article des recommandations du groupe de travail quant au développement de l'analyse de l'apprentissage dans les établissements d'enseignement avec l'objectif d'améliorer l'apprentissage. Certains éléments à considérer dans l'intégration de l'analyse de l'apprentissage, dont le rôle de différents acteurs (praticiens, chercheurs, décideurs, fournisseurs de services), les compétences nécessaires pour interpréter les données et les effets potentiels de telles analyses sur la conception de l'apprentissage, sont abordés.

Mots-clés : analyse de l'apprentissage ; learning analytics ; analytique de l'apprentissage ; éducation ; apprentissage en ligne

\begin{abstract}
At EDUsummIT2019, a working group reflected on the analysis of learning. As Frenchspeaking members of this group, this article discusses and addresses the recommendations of the working group for the development of learning analytics in educational institutions with the objective of improving learning. Some elements to consider in this perspective, including the role of the various players (practitioners, researchers, decision-makers, service providers), and the potential effects of such analysis on learning design, are addressed.
\end{abstract}

Keywords: Learning analysis; Learning analytics; Education; e-Learning

\section{Introduction}

Cet article vise à présenter un état de réflexions sur le thème des learning analytics (LA), basé sur les échanges qui ont eu lieu dans le cadre du groupe de travail (Team working group, 
TWG6) de l'EDUsummIT2019, nommé Putting learning back into learning analytics : optimizing learning through analyzing the data. En lien avec l'utilisation croissante des plateformes d'apprentissage, les learning analytics se taillent une place en éducation dans le travail des praticiens, mais aussi des décideurs politiques et des chercheurs, dans un contexte où l'enseignement et l'apprentissage doivent se poursuivre selon des modalités en mutation.

L'utilisation des technologies pour l'enseignement et l'apprentissage a pris un essor particulier depuis l'arrivée de la pandémie de COVID-19 et les mesures de confinement adoptées dans de nombreux pays. En éducation, des établissements, des enseignants et des élèves qui jusqu'ici n'avaient pas utilisé ces technologies, ont été incités à exploiter les outils et les ressources numériques afin de poursuivre leurs activités à distance et assurer ainsi une certaine continuité pédagogique ou éducative (Frau-Meigs, 2020). Les occasions de s'intéresser aux traces numériques et aux données concernant l'apprentissage se sont multipliées.

Notons d'abord que la traduction en français du terme learning analytics pose un certain défi : si le terme « analyse de l'apprentissage » est recommandé au Québec (Office québécois de la langue française, 2016), d'autres expressions sont utilisées dans d'autres pays francophones, par exemple « analytique des apprentissages numériques 》ou « science des données de l'apprentissage » (Labarthe \& Luengo, 2016) ou « analytique des activités d'apprentissage instrumentées »(Peraya, 2019). Dans la suite, nous utiliserons l'expression « analyse de l'apprentissage » pour learning analytics.

La première conférence internationale sur l'analyse et la connaissance de l'apprentissage a proposé la définition suivante : « L'analyse de l'apprentissage est la mesure, la collecte, l'analyse et la communication de données sur les apprenants et leurs contextes, dans le but de comprendre et d'optimiser l'apprentissage et les environnements dans lesquels il se produit ${ }^{1} \gg$ [traduction libre] (LAK, 2011). Comme l'ont précisé Long et Siemens (2011), l'analyse de l'apprentissage s'inscrit dans un contexte d'apprentissage (totalement ou partiellement) en ligne, où les données sont mises à profit pour appuyer ou orienter des décisions en éducation, que ce soit au niveau de la classe ou du département, ou pour l'analyse institutionnelle (academic analytics) au niveau régional, national ou international. Cet article s'inspirera de cette définition. Toutefois, ce n'est pas la seule définition de l'analyse de l'apprentissage qui a été proposée. Notons celle d'Ifenthaler (2015) : «L'utilisation d'informations statiques et dynamiques sur les apprenants et les environnements d'apprentissage, en les évaluant, en les obtenant et en les analysant, pour la modélisation en temps réel, la prédiction et l'optimisation des processus d'apprentissage, des environnements d'apprentissage, ainsi que de la prise de décision en éducation ${ }^{2} \gg$ [traduction libre].

Cet article s'intéressera à l'émergence de l'analyse de l'apprentissage dans le paysage éducatif, aux possibilités qui s'y rattachent ainsi qu'aux défis qu'elle soulève. Il situe d'abord le

\footnotetext{
${ }^{1}$ « Learning analytics is the measurement, collection, analysis and reporting of data about learners and their contexts, for purposes of understanding and optimising learning and the environments in which it occurs » (LAK, 2011).

2 «The use of static and dynamic information about learners and learning environments, assessing, eliciting and analysing it, for real-time modelling, prediction and optimization of learning processes, learning environments, as well as educational decision-making » (Ifenthaler, 2015).
} 
groupe de travail TWG6 dans la lignée des EDUsummIT précédents et indique ses principaux objectifs. Puis il présente les grandes lignes du domaine de l'analyse de l'apprentissage. Enfin les recommandations du groupe de travail sont rapportées et discutées.

\section{Le TWG6 d'EDUsummIT2019}

L'EDUsummIT (International summit on information technology in education) a lieu tous les deux ans. L'événement rassemble une communauté internationale de chercheurs, de décideurs et de pédagogues engagés à soutenir l'intégration efficace des technologies de l'information dans l'éducation en favorisant la diffusion active et l'utilisation de la recherche.

Lors de l'édition précédente, en 2017, deux groupes de travail avaient mentionné l'analyse de l'apprentissage comme piste d'action. Le groupe de travail Education systems in the digital age : The need for alignment avait souligné la nécessité de rassembler des traces et des preuves sur l'apprentissage et son contexte. Le groupe soulignait les défis éthiques de l'utilisation des données d'apprentissage ainsi que les enjeux de confidentialité (Butler et al., 2018). Deux ans plus tôt, le groupe Assessment as, for, and of Learning in the $21^{\text {st }}$ century avait recommandé de soutenir le développement de l'analyse de l'apprentissage, afin de développer les capacités d'évaluation et de rétroaction formatives (Spector et al., 2016).

En 2017, le groupe de travail Formative assessment supported by technology mentionnait quant à lui qu'il fallait encourager la création de possibilités de travail collaboratif dans la recherche en éducation afin d'examiner les liens complexes entre une collecte de données significatives, l'interprétation des données et l'utilisation des données pour soutenir les enseignants et les apprenants (Lai et al., 2017). Cette recommandation, qui invite à porter attention à la complexité et à la richesse de l'apprentissage, pavait la voie à l'émergence d'un groupe pour l'EDUsummIT suivant. Le TWG6 ${ }^{3}$ a ainsi été formé dans le cadre de 1'EDUsummIT2019 qui a eu lieu sous le thème Learners and learning contexts : New alignments for the digital age.

Ce groupe sur l'analyse de l'apprentissage s'est donné comme objectif principal d'examiner comment les chercheurs, les praticiens et les décideurs politiques peuvent contribuer à orienter les développements de l'analyse de l'apprentissage et de ses usages pour améliorer les processus d'apprentissage. Le groupe s'est aussi proposé d'examiner comment aider les différents intervenants à comprendre les méthodes et les résultats de l'analyse et à faire face à des conflits, jugés inévitables, entre des objectifs et des méthodes concurrentes d'utilisation des données dans un cadre éducatif. L'exploitation des données d'apprentissage, par exemple pour suivre les progrès des apprenants,

\footnotetext{
${ }^{3}$ Le groupe de travail intitulé Putting learning back into learning analytics : optimizing learning through analyzing the data (TW6) regroupait les membres suivants : Monique Baron (Sorbonne Université), Jill Downie (Curtin University), Sandra Elliott (Global Grid for Learning), David Gibson (Curtin University) (coresponsable), Dirk Ifenthaler (University of Mannheim) (coresponsable), Séverine Parent (Université du Québec à Rimouski), Doreen Prasse (The Schwyz University of Teacher Education), Jonathan San Diego (King's College London) (coresponsable), Atsushi Shimada (Kyushu University), Masanori Yamada (Kyushu University).
} 
épauler les enseignants, obtenir les informations nécessaires à la prise de décision et améliorer l'apprentissage, porte à la fois ses lots de promesses, de défis et de dérives possibles.

Sur la base d'un document de travail et de références proposés par les responsables du TWG6 ${ }^{4}$, un travail collaboratif de réflexion a été fait en amont de la rencontre par l'ensemble des membres du groupe, puis des discussions et une négociation ont eu lieu pendant l'événement, menant dans un premier temps à une liste de recommandations ${ }^{5}$. L'écriture collaborative a mené à différentes publications, dont une dans le livre électronique Learners and Learning Contexts. New Alignments for the Digital Age (Fisser \& Phillips, 2020) et une autre dans une revue (Ifenthaler et al., 2020). La suite de notre article permettra de porter un regard sur le domaine de l'analyse de l'apprentissage et de rapporter les résultats des discussions du groupe de travail agrémentés de quelques réflexions complémentaires.

\section{Le domaine de l'analyse de l'apprentissage}

Les avancées des technologies et de différents domaines de recherche ont contribué à la conception d'environnements informatiques pour l'apprentissage humain (EIAH), parmi lesquels, vers la fin des années 1990, les environnements numériques d'apprentissage (ENA) ${ }^{6}$. Les ENA regroupent des fonctionnalités qui soutiennent des activités d'enseignement et d'apprentissage, facilitant notamment la communication, le partage d'informations et de ressources numériques entre enseignants et étudiants, et parfois entre les étudiants. Ils ont soutenu le développement de formes d'apprentissage et d'enseignement entièrement ou partiellement en ligne, que la formation soit offerte en présence, à distance ou de manière hybride. Les traces d'activités dans une plateforme d'apprentissage peuvent être enregistrées, ce qui permet le recueil de données liées à l'interaction des apprenants (et des autres acteurs) avec le dispositif utilisé.

Des travaux de recherche ont été consacrés au début des années 2000 à la conception d'outils d'analyse de traces (Choquet et al., 2007), permettant notamment aux intervenants pédagogiques de suivre et de conseiller les apprenants utilisant des plateformes en ligne, d'analyser leurs comportements, pas à pas ou sur l'ensemble du cours, à une échelle individuelle ou collective (groupe ou classe, par exemple) et sur des durées variables (activité ou session, par exemple). Ces analyses de traces avaient notamment pour but de comprendre les comportements des apprenants afin de leur faire des retours appropriés, de les évaluer ou bien d'évaluer la conception du scénario d'apprentissage, ou encore d'analyser les usages pour un retour aux concepteurs de la plateforme.

\footnotetext{
${ }^{4}$ Le document de travail réalisé en amont de la rencontre est disponible en ligne : TWG6's Working document (19 septembre 2019).

${ }^{5}$ Le document des recommandations est disponible en ligne : TWG6's contribution to EDUsummIT 2019's Action Agenda for Advances in Education (13 octobre 2019).

${ }^{6}$ Un ENA est « un ensemble d'applications et de logiciels informatiques soutenant les activités liées à l'enseignement, la formation et l'apprentissage » (GTN-Québec, 2006 cité par Blanc et Guay, 2017). L'appellation en anglais est LMS (learning management systems). On utilise aussi l'expression «plateforme d'apprentissage ».
} 
C'est ainsi que s'est constitué le courant de recherche de l'analyse de l'apprentissage, dans le prolongement de travaux relatifs à la modélisation de l'apprenant (développés initialement dans le domaine des tuteurs intelligents) et de travaux relatifs à l'adaptation et à la personnalisation des environnements d'apprentissage (Lavoué \& Rinaudo, 2012). Ce courant, dont les principes généraux ont été présentés et discutés par Friesen (2013), s'est distingué de celui de l'exploration de données éducatives (educational data mining) lié au développement des méthodes de fouille de données et au recueil de données massives (big data) dans des plateformes d'apprentissage, qui visait plutôt à analyser les propriétés spécifiques des données éducatives (International Educational Data Mining Society). Ces deux courants partagent cependant certains objectifs et techniques d'investigation.

\section{Un domaine pluriel}

Comme l'indique le site de la Society for Learning Analytics Research (SoLAR, s.d.) :

L'analyse de l'apprentissage est à la fois un domaine académique et un marché commercial qui a pris forme rapidement au cours de la dernière décennie. En tant que domaine de recherche et d'enseignement, l'analyse de l'apprentissage se situe à la convergence de l'apprentissage (par exemple, recherche en éducation, sciences de l'apprentissage et de l'évaluation, technologie éducative), de l'analyse (par exemple, statistiques, visualisation, informatique/science des données, intelligence artificielle) et de la conception centrée sur l'humain (par exemple, utilisabilité, conception participative, réflexion sur les systèmes sociotechniques $)^{7}$. [traduction libre]

Les approches et les répercussions de l'analyse de l'apprentissage présentent un défi relativement nouveau, complexe et multidisciplinaire, qui exige que les parties prenantes (chercheurs, acteurs de l'éducation, ingénieurs, décideurs, acteurs du marché) travaillent ensemble, pour une variété d'objectifs qui peuvent être différents selon les points de vue des intervenants et susceptibles d'entraîner des conflits potentiels (Gibson \& Ifenthaler, 2017). Par exemple, dans le domaine de l'évaluation des apprentissages, le personnel enseignant et le personnel administratif n'ont pas nécessairement le même regard : pour certains, un jugement sommatif peut être suffisant, pour d'autres, un tel jugement ne suffit pas nécessairement à décrire une réalité jugée plus complexe. Selon SoLAR (s.d.), l'analyse de l'apprentissage vise globalement l'optimisation de l'apprentissage et des environnements dans lesquels il se déroule. Cependant, cette idée d'optimisation pourrait ne pas être la même pour tous les acteurs de l'éducation, notamment selon le niveau du système éducatif où ils interviennent.

\section{Les objectifs et méthodes}

Les chercheurs et praticiens qui se sont intéressés à l'analyse de l'apprentissage ont mis l'accent sur différentes finalités. Ainsi, pour Ifenthaler (2015), l'analyse de l'apprentissage vise la

\footnotetext{
${ }^{7}$ « Learning analytics is both an academic field and commercial marketplace which have taken rapid shape over the last decade. As a research and teaching field, Learning Analytics sits at the convergence of Learning (e.g. educational research, learning and assessment sciences, educational technology), Analytics (e.g. statistics, visualization, computer/data sciences, artificial intelligence), and Human-Centered Design (e.g. usability, participatory design, sociotechnical systems thinking) » : Society for Learning Analytics Research (SoLAR, s.d.).
} 
modélisation, la prévision et l'optimisation en temps réel des processus et des environnements d'apprentissage, ainsi que de la prise de décisions en éducation. Sur son site, SoLAR (s.d.) propose un classement en quatre types d'analyse, selon les objectifs et les méthodes utilisées : descriptive, diagnostique, prédictive et prescriptive ${ }^{8}$.

Parmi les objectifs de l'analyse de l'apprentissage, certains concernent particulièrement les apprenants en tant qu'agents actifs dans leur apprentissage (Gašević et al., 2015). Par exemple, dans certains collèges et universités, des systèmes d'alerte ont été mis en place pour déceler au plus tôt les étudiants dont la réussite scolaire est jugée incertaine. Ainsi, la Cité collégiale (Ontario) et l’Université Laval (Québec), au Canada, ont développé des systèmes qui informent les acteurs de l'éducation et les étudiants de telles situations, en visualisant un indicateur sous la forme de feux tricolores (Deschênes \& Parent, 2019). D'autres systèmes permettent aux apprenants de participer plus directement à l'analyse de l'apprentissage. Des systèmes, comme Fovéa, sondent les apprenants et collectent des données quantitatives et qualitatives au fil de la session (Parent \& Deschênes, 2018). D'autres approches placent l'apprenant au centre de ses apprentissages. Les recherches concernant le computer supported collaborative learning, qui prend en considération l'aspect social de l'apprentissage et l'utilisation de l'analyse de l'apprentissage, vont dans ce sens. Par exemple, le Knowledge Forum (KF), qui, depuis plusieurs années, permet aux apprenants de construire un discours collectif autour d'une problématique, rend disponibles des outils d'analyse du discours et d'analyse de la collaboration permettant à l'apprenant d'analyser sa participation (Teplovs et al., 2007).

\section{La communication des résultats de l'analyse de l'apprentissage}

Pour les usagers d'outils d'analyse de l'apprentissage, la communication des résultats est un élément important, tant sur le contenu que sur la forme, ainsi que sur le moment où elle a lieu. En direction des apprenants, des informations pourront être transmises par des rétroactions textuelles, des valeurs numériques (notes obtenues, temps passé sur les activités par exemple) ou des visualisations graphiques (comme le graphe du cheminement ou la courbe d'évolution des résultats), en temps réel ou en différé. Pour différents acteurs ont été développées des formes de visualisation analogues à celles utilisées dans d'autres secteurs de l'aide à la décision, notamment des tableaux de bord ${ }^{9}$ qui rassemblent des indicateurs jugés pertinents. Les tableaux de bord en éducation permettent de fournir divers types de commentaires aux enseignants et aux élèves, comme de cartographier l'acquisition de compétences ou d'indiquer la proportion d'élèves ayant réussi les objectifs d'apprentissage (Thille \& Zimmaro, 2017). Ainsi, avec les outils associés aux tableaux de bord permettant de collecter, d'analyser des données et de visualiser des informations, souvent en temps réel, de nouvelles possibilités émergent pour analyser les situations et prendre des décisions.

8 Des précisions concernant ces objectifs peuvent être consultées en ligne. Repéré à https://www.researchgate.net/publication/346260896_Methodologies_de_l'analyse_de_l'apprentissage (traduction de SoLAR).

${ }^{9}$ Un tableau de bord est un affichage visuel des informations les plus importantes nécessaires pour atteindre un ou plusieurs objectifs, consolidés et disposés sur un seul écran afin que les informations puissent être observées en un coup d'œil (Few, 2004). 
Cependant, comment s'assurer de la capacité des différents acteurs de l'éducation à interpréter et à utiliser, au fil de leurs activités, les informations ainsi communiquées?

\section{Les recommandations du TWG6}

Les travaux du TWG6 ont mené le groupe à proposer des recommandations ${ }^{10}$, sous la forme de six stratégies précisées chacune par deux actions principales, adressées aux décideurs politiques, aux chercheurs et aux praticiens, la moitié des recommandations concernant toutes les parties prenantes (Ifenthaler et al., 2020). Ces stratégies et actions sont présentées et discutées dans cette section en abordant à la fois les possibilités, mais aussi les défis qu'il reste à relever en matière d'analyse de l'apprentissage.

\section{Soutenir la pratique fondée sur des données probantes afin qu'elle soit conduite par l'analyse de l'apprentissage}

Pour soutenir la pratique informée par des données (evidence-based learning or teaching) afin qu'elle soit conduite par l'analyse de l'apprentissage, le TWG6 suggère, d'une part, d'élaborer des politiques axées sur le leadership, l'apprentissage professionnel, les mécanismes habilitants et la gouvernance des données, et, d'autre part, d'assurer un accès ouvert aux ressources et aux meilleures pratiques. Cette recommandation peut paver la voie à des constats et des initiatives novatrices. Cependant, il importe de considérer aussi les défis que la communauté de praticiens et de chercheurs n'a pas tout à fait terminé de relever.

Certains outils généraux d'analyse de l'apprentissage sont utilisés comme des tableaux de bord présentant sous différentes formes des résultats d'évaluations ou permettant le suivi de la réussite des étudiants. Cependant, même si l'utilisation de telles données pour guider des décisions s'inscrit dans une certaine continuité en éducation, l'utilisation des outils et leur intégration aux pratiques en ligne peuvent nécessiter un apprentissage pour les acteurs pédagogiques ou les responsables institutionnels. C'est aussi le cas pour des outils plus spécifiques (à une discipline, un niveau, un cursus ou une approche pédagogique, par exemple), qui peuvent nécessiter un développement professionnel comme une formation ou l'étude de ressources particulières, dont des guides de bonnes pratiques, qu'il convient de rendre facilement accessibles à tous les acteurs concernés.

Pour d'autres types d'outils ou d'approches, des modèles, méthodes et algorithmes concernant l'analyse, la représentation et l'usage de données éducatives font encore l'objet de recherches, comme en témoignent notamment les conférences annuelles LAK (learning analytics and knowledge) et EDM (educational data mining). Passer de résultats de recherche à la conception et la diffusion d'outils fiables, puis à leur utilisation éclairée, nécessite plusieurs étapes, ainsi que des réponses à diverses questions dont certaines seront évoquées dans les recommandations suivantes. Les chercheurs en sciences de l'éducation doivent en particulier approfondir les connaissances quant

\footnotetext{
${ }^{10}$ La version en français des recommandations, stratégies et actions du groupe de travail est disponible en ligne. Repéré https://www.researchgate.net/publication/346260923_Les_recommandations_strategies_et_actions_du_group e_de_travail_6_de_1\%27EDUsummIT_2019_TWG_6_Putting_learning_back_into_learning_analytics
} 
aux répercussions positives anticipées ou espérées sur l'apprentissage et quant à ce qui peut être considéré comme les bonnes (ou meilleures) pratiques.

Ainsi, des notions et outils d'analyse de l'apprentissage peuvent être introduits dès à présent dans la formation et la pratique des acteurs de l'éducation, mais il est souhaitable que de nouveaux développements et résultats soient élaborés par les chercheurs, avant que les décideurs puissent envisager des politiques générales de conduite de la pratique par l'analyse de l'apprentissage et « la gouvernance des données »avec un degré de confiance suffisant.

\section{Promouvoir l'adoption de l'analyse de l'apprentissage}

Parmi les actions pour promouvoir l'adoption de l'analyse de l'apprentissage et le développement d'outils, le TWG6 suggère d'élaborer des normes, des principes directeurs et des politiques, ainsi que de favoriser les meilleures pratiques d'utilisation de l'analyse de l'apprentissage. Il est aussi proposé de permettre une adaptation organisationnelle, en vue d'aider les parties prenantes à utiliser l'analyse de l'apprentissage pour soutenir l'apprentissage.

Du point de vue des fournisseurs de services, l'adoption de normes techniques et de principes directeurs permettant la standardisation et l'interopérabilité des outils en favoriserait la conception et la diffusion. Par exemple, la spécification Experience API (xAPI), conçue au début des années 2010, a eu pour objectif la description standardisée et l'échange des données d'expériences d'apprentissage (learning records) entre différents systèmes informatiques, permettant ainsi une certaine interopérabilité et le recueil de données au-delà des plateformes d'apprentissage proprement dites. Ces normes et principes directeurs pourraient contribuer à baliser une démarche qualité qui serait une garantie auprès des établissements et autres clients potentiels.

Le groupe de travail a mentionné également le besoin de transparence. Des enjeux de l'utilisation d'outils d'analyse de l'apprentissage, qu'elle soit soutenue par l'établissement d'enseignement ou par des fournisseurs de services, se situent au niveau de la compréhension des méthodes et des algorithmes utilisés. Les algorithmes et les modèles sous-jacents peuvent en effet être complexes ou difficiles d'accès. Est-ce que la façon d'utiliser les données d'apprentissage est transparente ou relève davantage d'une boîte noire ?

Si l'algorithme est accessible, par exemple dans le cas d'un algorithme dit libre (open source), cela ne garantit pas qu'il soit compréhensible. Pour les scientifiques des données (data scientists), il y a un enjeu d'explicabilité des indicateurs retenus et des décisions automatisées, voire l'obligation de rendre compte suivant un principe de transparence ou loyauté algorithmique (Castets-Renard, 2018). Fournir des explications et de la transparence en exposant le raisonnement et les données traitées est un enjeu bien documenté pour les systèmes de recommandations (Herlocker et al., 2000) et pour les systèmes utilisant des algorithmes d'apprentissage automatique, comme le mentionne le TWG4, groupe de travail sur l'apprentissage automatique (machine learning) à 1'EDUsummIT2019. En outre, pour ce dernier, des biais peuvent être intégrés, intentionnellement ou non, au niveau de l'algorithme ou des données d'entraînement; difficiles à détecter, ils peuvent mener à des décisions discutables, voire inéquitables (Webb et al., 2020). Des décisions prises sur la base de données massives et d'algorithmes complexes, qu'elles soient menées par de bonnes intentions ou par des considérations éloignées du terrain, peuvent avoir des conséquences négatives, voire désastreuses (O’Neil, 2016). C’est par exemple le cas de réformes éducatives qui visent à 
repérer les enseignants les moins performants sur la base des résultats de leurs élèves, ou encore dans le cas de l'évaluation des demandes d'accès aux études supérieures qui renforcent les inégalités en reproduisant certains biais.

La bonne compréhension des systèmes qui soutiennent l'analyse de l'apprentissage est une responsabilité partagée entre la personne ou l'organisme qui les conçoit et les utilisateurs. Les traitements effectués sur les données devraient être documentés par les concepteurs des algorithmes, avec des explications adaptées pour les différents types d'acteurs concernés. Ainsi un code de conduite est sans doute nécessaire afin de guider le développement d'outils pour l'analyse de l'apprentissage.

Du point de vue des utilisateurs, l'usage par les divers acteurs des outils d'analyse de données d'apprentissage suppose qu'ils comprennent suffisamment la nature des traitements effectués. Quelles compétences sont nécessaires pour utiliser ces outils, pour interpréter correctement les données, pour comprendre les algorithmes et pour apprécier la visualisation des données? Nous reviendrons sur ces questions au point « Influencer l'apprentissage par des outils d'analyse ».

\section{Informer et guider les fournisseurs de services de données et les utilisateurs}

Deux actions importantes sont proposées afin d'informer et de guider les fournisseurs de services de données et les utilisateurs : promouvoir une assurance qualité éthique, grâce notamment à des mécanismes tels que des normes, des processus d'accréditation, des audits et des recommandations, et promouvoir la durabilité et l'évolutivité des outils d'analyse de l'apprentissage.

Les considérations éthiques, notamment le respect de la vie privée, la sécurité et la propriété des données personnelles, le traitement loyal (fair use) des données, sont des préoccupations incontournables lors de la conduite de projets d'analyse de l'apprentissage. Les acteurs de l'éducation, incluant les apprenants, devraient être informés du type d'informations collectées, de la manière dont elles sont collectées, stockées et traitées (Pardo \& Siemens, 2014).

Le RGPD (Règlement général sur la protection des données), applicable en Europe depuis le 25 mai 2018, et le standard international ISO/IEC 27701 for privacy information management (publié en aout 2019) ont édicté des règles qui visent à la fois la protection des personnes concernées par un traitement de leurs données à caractère personnel et la responsabilisation des acteurs de ce traitement. Inspiré du modèle européen, le projet de loi nº 64 au Québec propose de moderniser des dispositions législatives en matière de protection des renseignements personnels. Dans le domaine de l'éducation en Europe, plusieurs de ces considérations ont fait l'objet de chartes éthiques, comme celle du projet HUBBLE ${ }^{11}$ ou du projet GAR (gestionnaire d'accès aux ressources) qui accompagne le développement des usages des ressources numériques dans les établissements d'enseignement afin de garantir la protection des données des acteurs pédagogiques.

Pardo et Siemens (2014) ont présenté quatre principes pour aborder les nombreux défis découlant de la gestion de données personnelles dans l'analyse de l'apprentissage : la transparence,

\footnotetext{
${ }^{11}$ L'objectif du projet HUBBLE (http://hubblelearn.imag.fr) était de créer un observatoire national français pour la construction et le partage de processus d'analyse de données éducatives massives, issues des traces laissées dans des environnements de type e-learning, en vue d'analyser et d'expliquer des phénomènes d'enseignement et d'apprentissage avec ces environnements.
} 
la sécurité, la responsabilité et le contrôle des étudiants sur les données. Les politiques de sécurité doivent clairement déterminer quels utilisateurs ont accès à quelles données et quels types d'opérations sont autorisées, et ce en considérant le compromis entre l'accès et l'avantage. Ainsi, des enseignants pourraient accéder à des informations concernant leurs étudiants, certaines étant anonymisées, pour offrir un portrait de groupe sans cibler des étudiants en particulier. La responsabilité est un principe qui se traduit par la robustesse et par la rigueur de l'ensemble du processus lié à l'analyse de l'apprentissage dans une organisation. Le principe du droit des utilisateurs permet aux acteurs de l'éducation d'accéder aux données obtenues à leur sujet et de les corriger. Pardo et Siemens (2014) soutiennent que le contrôle des acteurs sur les données est présent dans la plupart des règlementations en matière de confidentialité, mais que la manière dont il est mis en œuvre, notamment dans le privacy by design, varie considérablement. Ce principe, qui permet à un acteur de porter un regard critique sur les données collectées le concernant, est lié à celui de transparence, en ce sens que pour qu'il puisse contrôler les données collectées il doit savoir ce qui est collecté, quand, comment et comment les données sont traitées.

L'évolution des technologies, des supports, des normes techniques, tout autant que des besoins et des usages des acteurs pédagogiques, peut être jugée rapide. Il reste des questions à résoudre, comme l'anonymisation des données répondant à l'exigence de confidentialité (mais qui devrait néanmoins permettre, dans certains cas, de suivre les élèves dans la durée), la conservation, la prévision du stockage et des traitements à plus ou moins long terme des données recueillies, la propriété des données et la mise en œuvre du droit à l'oubli. Ces questions, auxquelles s'ajouteront probablement d'autres, devront être considérées par tous les acteurs, notamment les fournisseurs de services et les décideurs.

\section{Influencer l'apprentissage par des outils d'analyse}

Afin que l'analyse de l'apprentissage puisse bénéficier d'abord à l'apprentissage, le TWG6 propose, d'une part, de veiller à ce que tous les acteurs concernés acquièrent des niveaux de connaissances suffisants en science des données et, d'autre part, de fournir des outils d'analyse adaptés aux besoins des différentes parties prenantes, afin d'améliorer l'apprentissage. Les acteurs pourront ainsi s'assurer de comprendre les résultats de l'analyse et d'en tirer parti pour leurs activités.

Cette nouvelle littératie pourrait proposer des pistes de réponses à différentes questions liées à la compréhension de l'analyse de l'apprentissage. Quelles connaissances et compétences sont nécessaires pour comprendre les nouvelles méthodes de science des données et pour adapter celles-ci aux traditions qualitatives et quantitatives en éducation? Que doivent savoir les apprenants pour comprendre - et être des acteurs critiques de - l'utilisation de leurs données et des résultats des algorithmes d'analyse?

Certaines visualisations proposées par des tableaux de bord peuvent être difficiles à appréhender. Par exemple, les travaux de Tufte (2001) et plus récemment ceux de Cairo (2019), ont permis d'apprécier la rigueur des représentations graphiques, tant pour leur exactitude que pour leur optimisation. Cependant, Cairo (2019) mentionne que les graphiques sont des arguments visuels et qu'il est aisé de mal les interpréter. Il propose un regard critique sur les graphiques, notamment en s’intéressant à ce que le graphique ne montre pas et en se gardant de tirer des conclusions erronées 
des données illustrées. La capacité à comprendre les représentations graphiques a des répercussions sur la création de sens et sur les inférences ultérieures (Alhadad, 2018). Comment s'assurer de la capacité des acteurs de l'éducation à comprendre, à utiliser ou à présenter l'information sous forme visuelle (dessins, diagrammes, graphes, photographies, plans, etc.), et comment les former à cette aptitude qu'on nomme la graphicatie?

Concernant la disponibilité d'outils d'analyse adaptés aux parties prenantes, il convient de répondre aux besoins des différents types d'acteurs, selon leur position et leur point de vue sur l'apprentissage. Par exemple, un enseignant ayant une classe à distance sera intéressé par des données en temps réel sur les activités des élèves, afin d'interagir avec eux à bon escient, alors qu'un responsable d'établissement pourrait ne s'intéresser qu'à une vision globale des résultats des évaluations finales.

Comment s'assurer que les outils proposés répondent aux besoins des acteurs, notamment des élèves ou étudiants de différents âges et niveaux d'apprentissage, et évoluant dans des contextes variés? De nombreux travaux ont été consacrés aux thèmes de la modélisation de l'apprenant, de l'adaptation et de la personnalisation des environnements depuis plusieurs décennies. Comment s'assurer que certaines approches ne visent pas à définir un cheminement « idéal » d'apprentissage qui marginaliserait ceux qui s'en éloignent? Dans le soutien à la réussite des étudiants qui ont des caractéristiques ou un profil spécifiques, les étudiants à besoins particuliers ou ceux de première génération par exemple, quel est le sens de l'optimisation de l'apprentissage? Face à une réalité où le parcours scolaire atypique devient de plus en plus fréquent, comment tirer profit de l'analyse de l'apprentissage en incluant tous les profils d'apprenants? Le regard porté par un étudiant sur ses apprentissages pourrait aussi ne pas concorder avec l'analyse qui lui est présentée. Ces écarts, encore peu documentés, méritent sans doute qu'on s'y attarde.

Une meilleure connaissance par les acteurs des processus, méthodes et possibilités de l'analyse de l'apprentissage leur permettrait aussi de mieux exprimer leurs besoins d'outils spécifiques, par exemple dans un cadre de conception centrée utilisateur (user-centred design).

En outre, certaines limites des outils actuels sont à considérer. La plupart des analyses d'apprentissage évaluent des objectifs d'apprentissage prédéfinis et l'acquisition par les apprenants de connaissances et de compétences à un moment donné (Schwartz \& Arena, 2013), plutôt que des compétences de réflexion d'ordre supérieur essentielles à l'innovation (Zhang et Chen, 2016). De plus, le portrait tracé par les données s'apparente essentiellement à un coup de sonde ponctuel plutôt qu'à la variation dans le temps de données qui pourraient fluctuer (Parent \& Deschênes, 2018). En effet, comme le mentionne Friesen (2013), les besoins des étudiants et leurs façons d'apprendre sont complexes et varient dans le temps, de même que leurs interactions avec des ressources (en ligne ou non), et il n'est pas aisé de saisir les liens de causalité entre les traces d'activités et la performance académique.

Même si les données sur lesquelles est basée l'analyse de l'apprentissage peuvent être nombreuses, elles ne captent pas l'ensemble de la situation d'apprentissage, dans toute sa complexité et sa richesse, comme les activités qui se déroulent à l'extérieur des environnements numériques ou des données qui concernent, de près ou de loin, le contexte de l'apprentissage ou de l'apprenant, contexte social ou culturel par exemple. Que l'on vise à améliorer la réussite éducative ou la réussite 
scolaire, il convient que tous les acteurs soient conscients des limites possibles et des répercussions potentielles des outils utilisés, et par suite du regard ainsi porté sur l'apprentissage et sur l'apprenant.

\section{Tirer parti de la relation entre design pédagogique (learning design) et analyse de l'apprentissage}

Un sujet de discussion dans le TWG6 a concerné les relations entre l'analyse de l'apprentissage et le design pédagogique (learning design). Sur ce point, le TWG6 recommande, d'une part, d'utiliser l'analyse de l'apprentissage pour être informé sur ce qui sous-tend le design pédagogique, l'enseignement, l'apprentissage et l'évaluation. D'autre part, il préconise de favoriser la recherche multidisciplinaire et participative, notamment pour assurer la qualité et suivre le cycle de vie des environnements d'apprentissage.

Le design pédagogique et l'analyse de l'apprentissage semblent nécessairement liés. En effet, comment savoir quelles données sont significatives et comment les interpréter de manière pertinente, sans information sur le design pédagogique (intentions pédagogiques, activités, scénarios prévus)? Comment améliorer un scénario pédagogique ou des activités d'apprentissage sans retour (données d'observations ou d'évaluations) sur leur mise en œuvre?

La conception et la réalisation de systèmes d'apprentissage en ligne ont amené à élaborer des méthodes spécifiques d'ingénierie pédagogique, ingénierie située « à l'intersection du design pédagogique, du génie logiciel et de l'ingénierie cognitive »(Paquette, 2002, p. 107), combinant la conception de l'apprentissage et son implantation dans un dispositif informatique. Le design pédagogique implique la relation entre les acteurs, les activités et les environnements (Paquette, 2004). Dans un contexte où l'on utilise l'analyse de l'apprentissage en soutien au design pédagogique, des données peuvent indiquer comment l'enseignant, d'autres intervenants pédagogiques ou des pairs, peuvent contribuer au processus d'apprentissage. La collecte de ces données utiles au suivi des activités et à leur soutien, ainsi qu'à l'amélioration du design pédagogique est à prévoir, en partie au moins, dans le processus d'ingénierie pédagogique, comme le proposait Pernin (2007) dans un modèle du cycle de vie d'un scénario d'apprentissage.

Le potentiel de l'analyse de l'apprentissage serait amélioré en se référant au design pédagogique qui documente l'intention pédagogique (Lockyer et al., 2013). De plus, il semble que l'enseignant peut jouer un rôle crucial en apportant un éclairage sur le contexte d'apprentissage et d'enseignement dans l'interprétation de l'analyse et aussi dans la prise de décisions basée sur l'analyse (Bakharia et al., 2016).

Dans le courant de recherche relatif aux environnements d'apprentissage adaptatifs, l'idée générale est de prendre en compte des données relatives aux apprenants, obtenues par un processus de modélisation à partir de traces, afin d'adapter dynamiquement les activités, les ressources ou les parcours proposés, ou encore l'interface (Lavoué \& Rinaudo, 2012). On peut voir là une synergie dynamique entre conception et analyse de l'apprentissage, où les données analysées vont influencer en temps réel l'environnement d'apprentissage proposé à l'apprenant (Ifenthaler et al., 2018).

Des liens plus étroits pourraient être tissés entre l'analyse de l'apprentissage et la recherche en sciences de l'apprentissage, comme le suggère Reimann (2016). D'une part, le potentiel de l'analyse de l'apprentissage pourrait être davantage exploité par la recherche en sciences de l'apprentissage, et, d'autre part, l'analyse de l'apprentissage aurait intérêt à se rapprocher de la 
recherche fondamentale sur l'apprentissage. Une connexion entre ces deux domaines pourrait être réalisée dans le cadre du courant design-based research (Design-Based Research Collective, 2003; Sanchez \& Monod-Ansaldi, 2015). Le renforcement des liens entre analyse de l'apprentissage, design pédagogique et recherche en sciences de l'éducation, notamment dans le cadre de projets pluridisciplinaires de conception basée sur la recherche et centrée sur les utilisateurs est à promouvoir.

\section{Comprendre les répercussions de la combinaison de données de tous les secteurs sur les interactions avec les individus}

Il convient de réfléchir aux répercussions de la combinaison de données de différents secteurs sur les interactions avec les individus, avant d'exploiter de telles possibilités en émergence. Le groupe TWG6 a proposé d'assurer la confidentialité des données et la sécurité de l'interopérabilité, ainsi que de garantir que le contrôle et la propriété des données sont clairs, transparents et entre les mains de la personne qui en est l'objet.

En effet, d'une part, des données personnelles sont collectées dans différents secteurs (données de santé, socioéconomiques, comportementales, réseaux sociaux, etc.) et l'interopérabilité des différents systèmes de données progresse. Dans la perspective où de tels systèmes seraient accessibles et interconnectés, comment anticiper les conséquences de la combinaison de ces données avec les données d'apprentissage, pour les individus et les établissements d'enseignement?

D'autre part, les capacités de collecte et de traitement de données multimodales (image, voix, données sensorielles, etc.) pouvant être intégrées à la situation d'apprentissage se développent et font l'objet de recherches dans des domaines spécialisés (imagerie, analyse du discours, biologie, psychologie, etc.). De telles données pourraient enrichir la compréhension de l'apprentissage, à condition de disposer de modèles et de théories qui en permettent l'analyse et l'interprétation dans le contexte d'apprentissage visé.

Dans ces perspectives, il importe aussi de renforcer les liens entre analyse de l'apprentissage et recherche en sciences de l'apprentissage. Par ailleurs, il convient d'être vigilant afin d'assurer pour toutes ces données, notamment celles qui seraient obtenues par l'interconnexion de systèmes, les mêmes garanties de confidentialité, de transparence, de traitement loyal, de sécurité et de contrôle par les personnes concernées, conformément aux règles éthiques mentionnées précédemment - qui, actuellement, ne sont pas prises en considération de la même manière dans tous les pays.

\section{Discussion}

L'expérience inédite de la pandémie de COVID-19, avec les périodes de confinement vécues en 2020 dans plusieurs pays, a mis en évidence l'intérêt de dispositifs d'apprentissage en ligne, et le potentiel de l'analyse de l'apprentissage pour soutenir les activités de tous les acteurs concernés par l'apprentissage. Si certaines possibilités étaient présentes, des disparités dans l'accès aux technologies et dans leurs usages ont aussi été mises en évidence, actualisant le besoin de développer les connaissances et compétences de tous les acteurs pour des usages adéquats des technologies et en particulier des systèmes destinés à soutenir l'apprentissage. Des obstacles systémiques peuvent rendre difficile l'ajustement des pratiques pédagogiques aux possibilités offertes par les données, que 
ce soit la vision de la direction qui fait défaut, les occasions manquées d'utiliser des données ou l'accès limité à la technologie (Daniels et al., 2013).

Certains enseignants peuvent se sentir contraints d'utiliser les technologies disponibles. La prévalence accrue des logiciels d'analyse de l'apprentissage, souvent imposée, permet d'automatiser de nombreuses décisions de classe au détriment du rôle de décideur professionnel actif des enseignants (Laferrière et al., 2020). Cette utilisation d'outils, grand public ou pédagogiques, peut refléter un désir de suivre les tendances ou de répondre aux besoins ou aspirations d'un groupe plutôt qu'un choix réfléchi de soutenir une stratégie pédagogique adaptée au contexte. Quelles conséquences cela pourrait-il avoir sur l'organisation des systèmes éducatifs, les acteurs de l'éducation et les formations elles-mêmes? Le « possible » est-il toujours souhaitable?

Dans une vision où l'analyse de l'apprentissage soutient la réussite, il importe de s'intéresser aux humains qui génèrent ces traces et aux environnements dans lesquels l'apprentissage se produit. Ainsi, la conception des outils d'analyse de l'apprentissage gagnerait à impliquer davantage les apprenants et les enseignants (conception centrée utilisateur ou user-centred design), de même que la documentation par la recherche basée sur le design (design-based research). Les alignements émergeant de l'analyse de l'apprentissage devront sans doute tenir compte des initiatives à grande échelle qui utilisent un grand nombre de données et de moyens pour comprendre l'apprentissage, mais aussi des initiatives plus locales et situées. Ces deux réalités gagnent à s'interféconder.

De plus, en matière d'analyse de l'apprentissage, des considérations qui dépassent le cadre de l'éducation pourraient retenir notre attention dans les prochaines années. En particulier, la question du développement durable soulève plusieurs interrogations. Dans quelle mesure une généralisation de l'analyse de l'apprentissage et de ses outils, associée à un développement plus important de l'apprentissage en ligne, sera-t-elle soutenable, non seulement pour les acteurs humains, mais aussi pour les systèmes technologiques et pour la planète? Quelles quantités de ressources (matérielles, mémoire, énergie, etc.) seront nécessaires au fonctionnement de tels dispositifs, au stockage et aux traitements des données, et quels impacts écologiques sont prévisibles?

La réflexion pourrait aussi s'inscrire dans le cadre plus général du développement et du déploiement des technologies numériques et de l'intelligence artificielle, pour lesquels divers comités d'éthique ont été constitués, dans des organismes scientifiques notamment. Parmi d'autres initiatives, la Déclaration de Montréal (Dilhac et al., 2018), issue d'un processus délibératif inclusif, propose un cadre éthique, des principes et des recommandations en vue d'orienter une transition numérique au bénéfice de tous et du bien commun. On y retrouve, à un niveau plus général, des règles éthiques et des recommandations que nous avons présentées et discutées dans cet article pour l'analyse de l'apprentissage, par exemple en matière de protection et d'exploitation des données personnelles, de transparence algorithmique, de littératie numérique et d'inclusion de la diversité. L'accent est mis aussi sur d'autres principes, comme le bien-être, le respect de l'autonomie, la solidarité, l'équité, la prudence, qui pourraient également orienter les suites de la réflexion pour ce qui concerne l'analyse de l'apprentissage. 


\section{Conclusion}

Les réflexions de l'édition de l'EDUsummIT2019 sur les apprenants et leurs contextes d'apprentissage ont permis d'aborder des alignements, ainsi que des décalages, concernant l'utilisation des technologies en éducation. Les recommandations faites par le groupe de travail TWG6 visent à guider les actions des décideurs, des chercheurs et des praticiens. Elles sont interreliées : connaissance et prise de conscience, principes directeurs, normes d'utilisation éthique et objectifs d'amélioration de l'apprentissage.

Le besoin de recherches pluridisciplinaires, combinant en particulier analyse de l'apprentissage, design pédagogique et sciences de l'éducation, selon des approches basées sur la conception et centrées utilisateurs et sur le design, a été souligné pour viser l'amélioration de l'apprentissage. Au fur et à mesure que de nouvelles méthodes et modèles d'analyse et de représentation des données seront créés et utilisés, il est essentiel que les parties prenantes soient soutenues pour en prendre connaissance, afin de mieux les comprendre et de s'approprier les outils qui les mettent en œuvre, pour pouvoir agir en conséquence.

Nous espérons avoir fourni des informations et des idées pour éclairer les réflexions, considérant qu'étant à ses débuts la pratique en analyse de l'apprentissage nourrit étroitement la recherche et vice-versa.

L'utilisation de ces données gagnera sans doute en importance dans le contexte actuel où les activités d'apprentissage en ligne sont encouragées, voire imposées, alors que les établissements d'enseignement sont partiellement ou entièrement inaccessibles, en raison de la pandémie. Ces possibilités de développement doivent inciter à renforcer la vigilance pour assurer la confidentialité, l'utilisation éthique, le contrôle par les personnes concernées de toutes les données personnelles utilisées par l'analyse de l'apprentissage. Des enjeux sont présents et des défis, connus actuellement ou qui émergeront, restent à relever. 


\section{Références}

Alhadad, S. S. (2018). Visualizing data to support judgement, inference, and decision making in learning analytics : Insights from cognitive psychology and visualization science. Journal of Learning Analytics, 5(2), 60-85. https://doi.org/10.18608/jla.2018.52.5

Bakharia, A., Corrin, L., de Barba, P., Kennedy, G., Gašević, D., Mulder, R., Williams, D., Dawson, S., \& Lockyer, L. (2016). A conceptual framework linking learning design with learning analytics. Dans D. Gašević (dir.), Proceedings of the Sixth International Conference on Learning Analytics \& Knowledge (p. 329-338). https://doi.org/10.1145/2883851.2883944

Butler, D., Leahy, M., Twining, P., Akoh, B., Chtouki, Y., Farshadnia, S., Moore, K., Nikolov, R., Pascual, C., Sherman, B., \& Valtonen, T. (2018). Education systems in the digital age : The need for alignment. Technology, Knowledge and Learning, 23(3), 473-494. https://doi.org/10.1007/s10758-018-9388-6

Cairo, A. (2019). Graphics that seem clear can easily be misread : Misreading data visualizations can reinforce biased perceptions. Scientific American.

https://www.scientificamerican.com/article/graphics-that-seem-clear-can-easily-be-misread/

Castets-Renard, C. (2018). Régulation des algorithmes et gouvernance du machine learning : vers une transparence et « explicabilité » des décisions algorithmiques? Revue Droit \& Affaires, Revue Paris II Assas, $15^{e}$ édition, 2018. https://ssrn.com/abstract=3391282

Choquet, C., Delozanne, E., \& Luengo, V. (2007). Éditorial du numéro spécial Analyse des traces d'utilisation dans les EIAH. Revue STICEF, 14. http://sticef.univlemans.fr/num/vol2007/sticef 2007 editoTrace.htm

Daniels, J., Jacobsen, M., Varnhagen, S., \& Friesen, S. (2013). Barriers to systemic, effective, and sustainable technology use in high school classrooms/Obstacles à l'utilisation systémique, efficace et durable de la technologie dans les salles de classe des écoles secondaires. La Revue canadienne de l'apprentissage et de la technologie, 39(4). https://doi.org/10.21432/T2SG67

Deschênes, M., \& Parent, S. (2019). (28 janvier 2019). Des outils d'analyse de l'apprentissage. Vitrine technologie-éducation. https://www.vteducation.org/fr/articles/analyse-delapprentissage/des-outils-danalyse-de-lapprentissage

Design-Based Research Collective. (2003). Design-based research : An emerging paradigm for educational inquiry. Educational Researcher, 32(1), 5-8. https://doi.org/10.3102/0013189X032001005

Dilhac, M. A., Abrassart, C., \& Voarino, N. (2018). Rapport de la Déclaration de Montréal pour un développement responsable de l'intelligence artificielle. https://www.declarationmontrealiaresponsable.com/la-declaration

Fisser, P., \& Phillips, M. (2020). Learners and learning contexts: New alignments for the digital age. https://edusummit2019.fse.ulaval.ca/files/edusummit2019 ebook.pdf 
Frau-Meigs, D. (2020). Pédagogie à distance : les enseignements du e-confinement.

The Conversation. http://theconversation.com/pedagogie-a-distance-les-enseignements-du-econfinement-137327

Friesen, N. (2013). Learning analytics : Readiness and rewards/L'analyse de l'apprentissage : état de préparation et récompenses. La Revue canadienne de l'apprentissage et de la technologie, 39(4). https://doi.org/10.21432/T2J01B

Gašević, D., Dawson, S., \& Siemens, G. (2015). Let's not forget: Learning analytics are about learning. TechTrends, 59(1), 64-71. https://link.springer.com/content/pdf/10.1007/s11528-0140822-x.pdf

Gibson, D., \& Ifenthaler, D. (2017). Preparing the next generation of education researchers for big data in higher education. Dans B. Daniel (dir.), Big Data and Learning Analytics in Higher Education (p. 29-42). Cham : Springer International Publishing.

Herlocker, J., Konstan, J. A., \& Riedl, J. (2002). An empirical analysis of design choices in neighborhood-based collaborative filtering algorithms. Information retrieval, 5(4), 287-310.

Ifenthaler, D. (2015). Learning analytics. Dans J. M. Spector (dir.), The SAGE Encyclopedia of Educational Technology, 2, 447-451. Thousand Oaks, CA : Sage.

Ifenthaler, D., Gibson, D., \& Dobozy, E. (2018). Informing learning design through analytics : Applying network graph analysis. Australasian Journal of Educational Technology, 34(2). https://doi.org/10.14742/ajet.3767

Ifenthaler, D., Gibson, D., Prasse, D., Shimada, A., \& Yamada, M. (2020). Putting learning back into learning analytics : Actions for policy makers, researchers, and practitioners. Educational Technology Research and Development, 1-20.

Labarthe, H., \& Luengo, V. (2016). L'analytique des apprentissages numériques (rapport de recherche). Paris : Laboratoire d'informatique de Paris 6 (LIP6). https://hal.archivesouvertes.fr/hal-01714229/document

Laferrière, T., Cox, M., \& Baron, G. -L. (2020). Résultats de l’EDUsummIT2019 au service de l'innovation pour l'éducation formelle des jeunes. Revue Adjectif, 1. http://www.epi.asso.fr/revue/articles/a2005d.htm

Lai, K. W., Voogt, J., \& Knezek, G. (2017). Rethinking learning in a digital age : EDUsummIT 2017 Summary Reports.

https://edusummit2019.fse.ulaval.ca/sites/edusummit2019.fse.ulaval.ca/files/edusummit 2017 ebook final 12.4.18.pdf

LAK. (27 février - $1^{\mathrm{er}}$ mars 2011). First International Conference on Learning Analytics and Knowledge. Banff, Alberta.

http://www.wikicfp.com/cfp/servlet/event.showcfp?eventid=11606

Lavoué, É., \& Rinaudo, J. -L. (2012). Éditorial du numéro spécial Individualisation, personnalisation et adaptation des EIAH. Revue STICEF, 19. https://www.persee.fr/doc/stice 1952-

$8302 \quad 2012$ num $19 \quad 1 \quad 1043$ 
Lockyer, L., Heathcote, E., \& Dawson, S. (2013). Informing pedagogical action: Aligning learning analytics with learning design. American Behavioral Scientist, 57(10), 1439-1459. http://doi.org/10.1177/0002764213479367

Long, P. D., \& Siemens, G. (2011). Penetrating the fog : Analytics in learning and education. Educause Review, 46(5), 31-40. https://er.educause.edu/articles/2011/9/penetrating-the-foganalytics-in-learning-and-education

O’Neil, C. (2016). Weapons of Math Destruction. How Big Data Increases Inequality and Threatens Democracy. New York, NY : Broadway Books.

Office québécois de la langue française (2016). Analyse de l'apprentissage. http://gdt.oqlf.gouv.qc.ca/ficheOqlf.aspx?Id Fiche=26541629

Paquette, G. (2002). L'ingénierie pédagogique. Pour construire l'apprentissage en réseau. Québec, QC : Presses de l'Université du Québec.

Pardo, A., \& Siemens, G. (2014). Ethical and privacy principles for learning analytics. British Journal of Educational Technology. https://doi.org/10.1111/bjet.12152

Parent, S., \& Deschênes, M. (juin 2018). Fovéa : un objet-frontière pour mieux comprendre l'expérience d'apprentissage des étudiants. Affiche présentée au Congrès annuel de la Société pour l'avancement de la pédagogie dans l'enseignement supérieur (SAPES), Sherbrooke, Québec.

Peraya, D. (2019). Les learning analytics en question. Distances et médiations des savoirs, 25. https://doi.org/10.4000/dms.3485

Pernin, J. -P. (2007). Mieux articuler activité pour l'apprentissage, artefacts logiciels et connaissances : vers un modèle d'ingénierie centré sur les scénarios. Dans M. Baron, D. Guin et L. Trouche (dir.), Environnements informatisés et ressources numériques pour l'apprentissage : conception et usages, regards croisés (p. 161-194). Cachan : Hermes Sciences-Lavoisier.

Reimann, P. (2016). Connecting learning analytics with learning research : The role of design-based research. Learning : Research and Practice, 2(2), 130-142.

https://doi.org/10.1080/23735082.2016.1210198

Sanchez, É., \& Monod-Ansaldi, R. (2015). Recherche collaborative orientée par la conception. Éducation \& didactique, 9(2), 73-94.

Schwartz, D. L., \& Arena, D. (2013). Measuring what Matters Most: Choice-based Assessments for the Digital Age. Cambridge, MA : The MIT Press.

SoLAR. (s.d.). What is Learning Analytics? https://www.solaresearch.org/about/what-is-learninganalytics/

Spector, M. J., Ifenthaler, D., Samspon, D., Yang, L., Mukama, E., Warusavitarana, A., Dona, K. L., Eichhorn, K., Fluck, A., Huang, R., Bridges, S., Lu, J., Ren, Y., Gui, X., Deneen, C. C., San Diego, J., \& Gibson, D. C. (2016). Technology enhanced formative assessment for $21^{\text {st }}$ century learning. Educational Technology \& Society, 19(3), 58-71. 
Teplovs, C., Donoahue, Z., Scardamalia, M., \& Philip, D. (2007, juillet). Tools for concurrent, embedded, and transformative assessment of knowledge building processes and progress. Dans C. A. Chinn, G. Erkens et S. Puntambekar (dir.), Proceedings of the 8th international conference on Computer supported collaborative learning (p. 721-723). https://dl.acm.org/doi/abs/10.5555/1599600.1599732

Thille, C., \& Zimmaro, D. (2017). Incorporating learning analytics in the classroom. New Directions for Higher Education, 179, 19-31. https://doi:10.1002/he.20240

Tufte, E. R. (2001). The Visual Display of Quantitative Information (vol. 2). Cheshire, CT: Graphics Press.

Webb, M. E., Fluck, A., Magenheim, J., Malyn-Smith, J., Waters, J., Deschênes, M., \& Zagami, J. (2020). Machine learning for human learners : Opportunities, issues, tensions and threats. Education Technology Research Development. https://doi.org/10.1007/s11423-020-09858-2

Zhang, J., \& Chen, B. (2016). Analytics for knowledge creation : Towards epistemic agency and design-mode thinking. Journal of Learning Analytics, 3(2), 139-163.

https://doi.org/10.18608/jla.2016.32.7 


\section{Authors}

Séverine Parent, Professeure en technologie éducative et littératie numérique, Université du Québec à Rimouski - Campus de Lévis, 1595, boulevard Alphonse-Desjardins, Lévis (Québec) G6V 0A6 Canada. Séverine Parent est professeure en technologie éducative et en littératie numérique. Ses champs de recherche concernent les technologies éducatives, notamment la compétence numérique et l'utilisation des données d'apprentissage, plus particulièrement la variation de l'engagement en contexte d'innovation. Dans sa pratique pédagogique, elle s'intéresse au renouvèlement des espaces d'apprentissage. Email : severine parent@uqar.ca

Monique Baron, Maître de conférence retraitée (Collaboratrice bénévole), Sorbonne Université, CNRS, LIP6, F-75005 Paris, France. Monique Baron est maître de conférences bénévole en informatique (retraitée). Investie dès les années 80 dans le domaine « IA et éducation », elle a contribué à constituer la communauté française de recherche en EIAH (Environnements Informatiques pour l'Apprentissage Humain). Ses principaux thèmes de recherche concernent la représentation des connaissances et la formalisation de raisonnements, notamment pour la modélisation de 1'apprenant. Email : Monique.Baron@1lip6.fr 